\title{
Description of the stability of residual marine fuel using ternary phase diagrams and SARA analysis
}

\author{
K.I. Smyshlyaeva, N.K. Kondrasheva, and V.A. Rudko \\ Saint-Petersburg Mining University, St. Petersburg, Russia
}

\begin{abstract}
The results of the analysis of the properties of components of residual marine fuels (RMF) are described. The stability areas of marine fuels are determined using ternary phase diagrams VisRes - ULSD LGOCC, Asphalt - ULSD - LCGO, Asphalt - ULSD - LGODC. The graphic method for determining the stability of A.B. Stankiewicz based on SARA analysis is used to describe the stability of RMF on the basis of VisRes - ULSD - LGOCC. The areas of stability, instability, and metastability of marine fuel are presented on the graph according to the Stankiewicz method, which can be used to predict the stability of RMF.
\end{abstract}

\section{Introduction}

The main international document regulating the quality of marine fuel today is ISO 8217:2017 "Marine fuels. Specifications", which includes requirements for 4 grades of distillate and 11 grades of residual fuel. Marine fuel is divided into two types: distillate fuel and residual fuel. Residual marine fuel (RMF) is used by vessels with medium-speed and low-speed engines. It is obtained on the basis of residual fractions with the addition of middle distillates.

The relevance of the production of low-sulfur stable residual marine fuel is due to environmental and economic challenges in the processing of raw materials both in the Russian Federation and around the world [1,2].

Stable marine fuel is a fuel that does not form a precipitate, does not delaminate, has a constant composition and properties during storage and use. The fuel obtained by the primary distillation of oil without the use of secondary processes is stable. However, not only straight-run fuel can be stable, but also a hydrocarbon mixture obtained on the basis of fractions of secondary oil refining processes. If the fuel components are incompatible, sediment will form and precipitate, and the fuel becomes unstable $[3,4]$. Therefore, special attention must be paid to the stability of the fuel system in the preparation of compounded residual marine fuels based on residual fractions. RMFs obtained by compounding residual and distillate oil fractions contains asphaltenes along with naphthenes, arenes, paraffin hydrocarbons. Asphaltenes can be the coagulation centers and cause sedimentation. The stability of these colloidal fuel systems is determined by the external molecular structure of asphaltenes, namely, the size of the solvate shell. The solvate shell is a structural and mechanical barrier that prevents the coagulation of asphaltenes [5-7]. 
Asphaltenes act as coagulation centers for macromolecular compounds. Due to their polarity, asphaltene molecules form associates, i.e. micelle nuclei. The outer layer is aromatic hydrocarbons, which are surface-active, so they are easily adsorbed on asphaltene micelles. Shells formed around asphaltenes prevent the micelles from merging into larger ones and precipitating. In addition, asphaltenes of thermo-destructive processes have lower aggregative stability in the colloidal system than asphaltenes of natural origin. Since asphaltenes of secondary origin formed during thermolysis have a less complex composition and molecular structure and less branching, they have lower solubility compared to natural asphaltenes [8-12].

Changing the composition of the fuel system by mixing stable residual fuel with a lowviscosity paraffin fraction can affect the stability of asphaltenes and make the entire fuel mixture unstable. When this happens, it means that the two fuels are incompatible. But with an increase in the number of aromatic hydrocarbons, asphaltenes stabilize, and stable fuel systems are formed. In this regard, there is a need to regulate the aggregate stability by adjusting the composition of the residual marine fuel [13-15].

\section{Experimental}

\subsection{Materials}

As objects of study, industrial products obtained from a mixture of West Siberian oils (Russian Federation) were selected, namely:

- Residual fractions (asphaltene carriers): visbroken residue (VisRes) from the visbreaking unit, asphalt from the deasphalting unit

- Medium disilute fractions (aromatic hydrocarbon carriers): light gas oil catalytic cracking (light cycle gasoil (LCGO) - light gas oil catalytic creaking (LGOCC in Russian) from a catalytic cracking unit (fluid catalytic creaking) (FCC), light gas oil delayed coking (LGODC) with delayed coking unit

- Medium disilute fraction (carrier of paraffin hydrocarbons) hydrotreated diesel fraction (ultra-low sulfur diesel) (ULSD) from a diesel hydrotreatment unit.

Each of the listed types of oil products was used to create model mixtures of residual marine fuel and was the predominant carrier of hydrocarbons of a certain type.

\subsection{Method}

The total sediment after aging (total sediment aged) is the sum of organic and inorganic substances insoluble in a paraffin solvent after preliminary aging of the sample, which are separated from the sample by filtering it.

In accordance with ISO 8217:2017, the value of this indicator for residual marine fuels should not exceed $0.1 \%$ wt. The stability determination of the prepared model mixtures of marine residual fuels was carried out in accordance with ISO 10307-2:2009 Petroleum products - Total sediment in residual fuel oils - Part 2: Determination using standard procedures for ageing.

The Stankiewicz graphical stability criterion is based on determining the ratio of saturates, aromatic hydrocarbons, resins and asphaltenes (SARA analysis); then the ratio of saturates / aromatic hydrocarbons ([S] / [Ar]) along the ordinate axis and the ratio of asphaltenes / resins ([A] / [R]) along the abscissa axis are plotted onto the graph. Two parts of the graph are clearly marked as stable and unstable regions, separated by a metastability region (curve) [16]. 


\section{Results and discussion}

Initially, the physicochemical properties and hydrocarbon composition (SARA analysis) of the following oil products were determined: visbreaker residue (VisRes), asphalt, light gas oil delayed coking and light gas oil catalytic creaking (LGODC and LGOCC), hydrotreated straight-run diesel fraction (HSRDF). The results are presented in Table 1.

Table 1. Properties of oil products.

\begin{tabular}{lccccc}
\hline \multicolumn{1}{c}{ Parameter } & \multicolumn{2}{c}{ Bottom fraction } & \multicolumn{3}{c}{ Middle distillate } \\
\cline { 2 - 6 } & VisRes & Asphalt & LGOCC & LGODC & ULSD \\
\hline Kinematic viscosity at $40{ }^{\circ} \mathrm{C}(\mathrm{mm} 2 / \mathrm{s})$ & - & - & 2.425 & 2.610 & 2.900 \\
Viscosity at $80{ }^{\circ} \mathrm{C}, \mathrm{sec}$. & 14.6 & 391.0 & - & - & - \\
Density at $15{ }^{\circ} \mathrm{C}(\mathrm{kg} / \mathrm{m} \mathrm{3})$ & 992.5 & 1027.2 & 956.0 & 856.0 & 835.8 \\
Sulfur, \% wt. & 0.9458 & 1.6000 & 0.0766 & 0.6590 & 0.0007 \\
Flash point in a closed cup, ${ }^{\circ} \mathrm{C}$ & - & - & 88 & 66 & 80 \\
Flash point in an open cup, ${ }^{\circ} \mathrm{C}$ & 174 & $>344$ & - & - & - \\
Pour point, ${ }^{\circ} \mathrm{C}$ & +15 & +61 & $<-60$ & -22 & -16 \\
Fractional composition, ${ }^{\circ} \mathrm{C}$ & & & & & \\
IBP & 350 & 457 & 197 & 180 & 200 \\
$10 \%$ & - & 532 & 210 & 193 & 215 \\
$50 \%$ & - & - & 259 & 247 & 282 \\
$90 \%$ & - & - & 298 & 343 & 343 \\
\hline FBP & 500 & 543 & 311 & 360 & 360 \\
paraffin-naphthene incl.: & 20.40 & 8.70 & 6.53 & 46.49 & 96.08 \\
aromatics & - & - & 0.56 & 29.24 & - \\
nydrocmal paraffins & 42.60 & 59.70 & 75.23 & 37.52 & 3.92 \\
olefins & - & - & 18.24 & 15.99 & - \\
resines & 11.70 & 28.40 & - & - & - \\
\hline asphaltenes & 25.30 & 3.20 & - & - & - \\
\hline
\end{tabular}

\subsection{The ternary phase diagram for residual marine fuel VisRes - ULSD - LGOCC}

The first step was to develop a ternary phase diagram (Figure 1), described in [17].

The following components were taken as basic components for the preparation of residual marine fuel samples: visbreaker residue (asphaltene carrier), light gas oil catalytic creaking (aromatic hydrocarbon carrier), hydrotreated straight-run diesel fraction (paraffin carrier).

To describe the stability of fuel systems based on three-component mixtures, samples of residual marine fuels with different component ratios with a step of $10 \%$ wt. were prepared and analyzed. The points corresponding to the pure components are located at the vertices of the triangle. On the sides of the triple phase diagram are samples consisting of two components. The points inside the triangle correspond to mixtures of the three components.

For all samples obtained, the total precipitate after aging was determined in accordance with ISO 10307-2:2009. Red color indicates samples that have a total precipitate after aging of more than $0.1 \%$ wt. These samples do not meet the requirements of ISO 8217:2017 in terms of the sediment after aging. This fuel will be unstable and will form a precipitate during storage. Green color indicates samples that have a total precipitate after aging of less than $0.1 \%$ wt. These samples comply with the requirements of ISO 8217:2017. This fuel will be stable, will not delaminate nor form a precipitate during storage. 


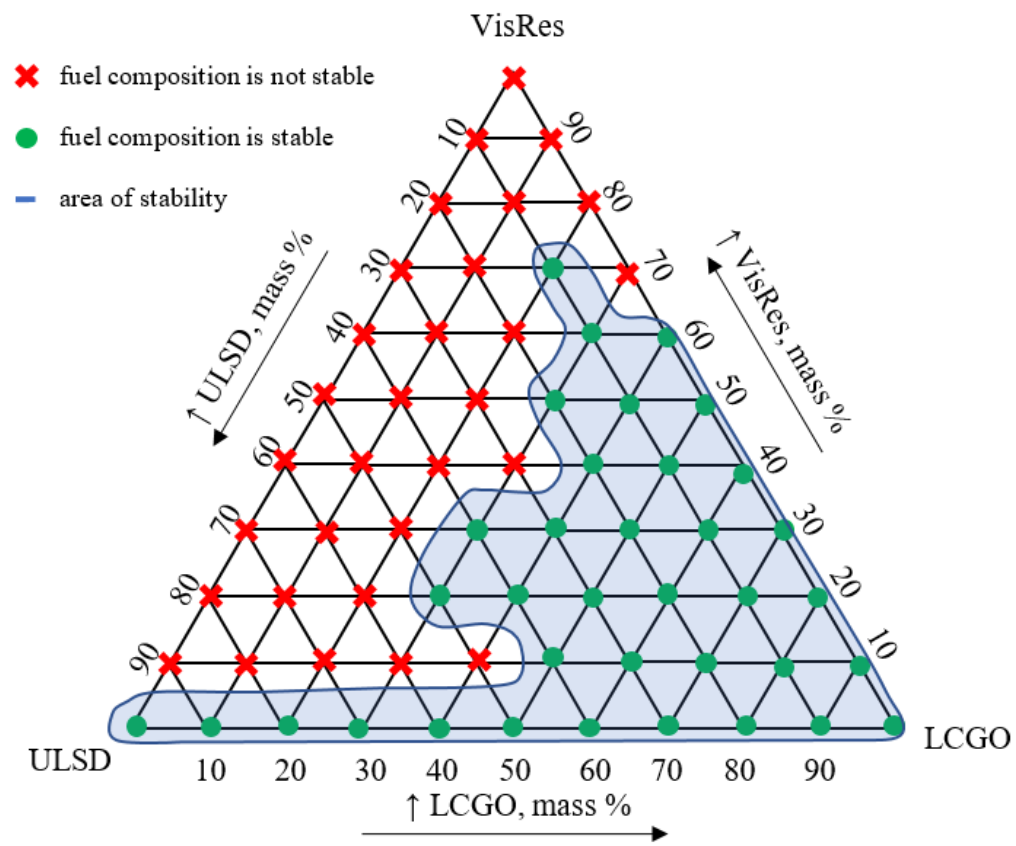

Fig. 1. The ternary phase diagram of the stability of the residual marine fuel VisRes - ULSD LCGO.

Residual marine fuel, consisting of VisRes, ULSD, LCGO, has a large area of instability, since VisRes contains $25.3 \%$ wt. of asphaltenes. VisRes is the residual fraction of the thermodestructive process. Asphaltenes of secondary origin have a less complex composition and molecular structure, less branching and are more prone to sedimentation. They have lower solubility compared to natural asphaltenes.

Hydrotreated straight-run diesel fraction contains $92.08 \%$ wt. of paraffin-naphthenic hydrocarbons. When compounding the pure components of ULSD (with a predominance of paraffin-naphthenic hydrocarbons) with VisRes (with a high content of asphaltenes), almost all samples totally precipitate after aging more than $0.1 \% \mathrm{wt}$, which is a characteristic of the instability of residual marine fuel.

When the hydrocarbon composition is changed, i.e., aromatic hydrocarbon carrier component is added to the system (LCGO contains $75.23 \% \mathrm{wt}$. of arenes), the value of the total precipitate after aging for the samples decreases to $0.1 \%$ wt. and less, which meets the requirements of ISO 8217: 2017 and characterizes the stability of the samples.

When a fraction carrier of aromatic hydrocarbons is introduced into the system, a transition to the stability region occurs, since the solubility of the particles of the dispersed phase of asphaltenes in a dispersion medium increases with an increase in the content of aromatic hydrocarbons in the dispersion medium, and decreases with an increase in paraffin-naphthenic hydrocarbons.

Thus, when compounding residual fractions containing asphaltenes (VisRes) with distillate fractions with a predominance of paraffin-naphthenic hydrocarbons (ULSD), asphaltene associates are combined, enlarged, and precipitated. When added to the residual fraction (VisRes) containing asphaltenes, i.e., aromatic hydrocarbons (LCGO), the stability of the fuel mixture increases. 


\subsection{The ternary phase diagram for residual marine fuel Asphalt - ULSD - LCGO}

In the second ternary phase diagram (Figure 2), the asphaltene carrier component is asphalt. Asphalt is a product of propane deasphalting of vacuum residue. The remaining two components, i.e., the carrier of paraffin-naphthenic hydrocarbons and the carrier of aromatic hydrocarbons, remained unchanged.

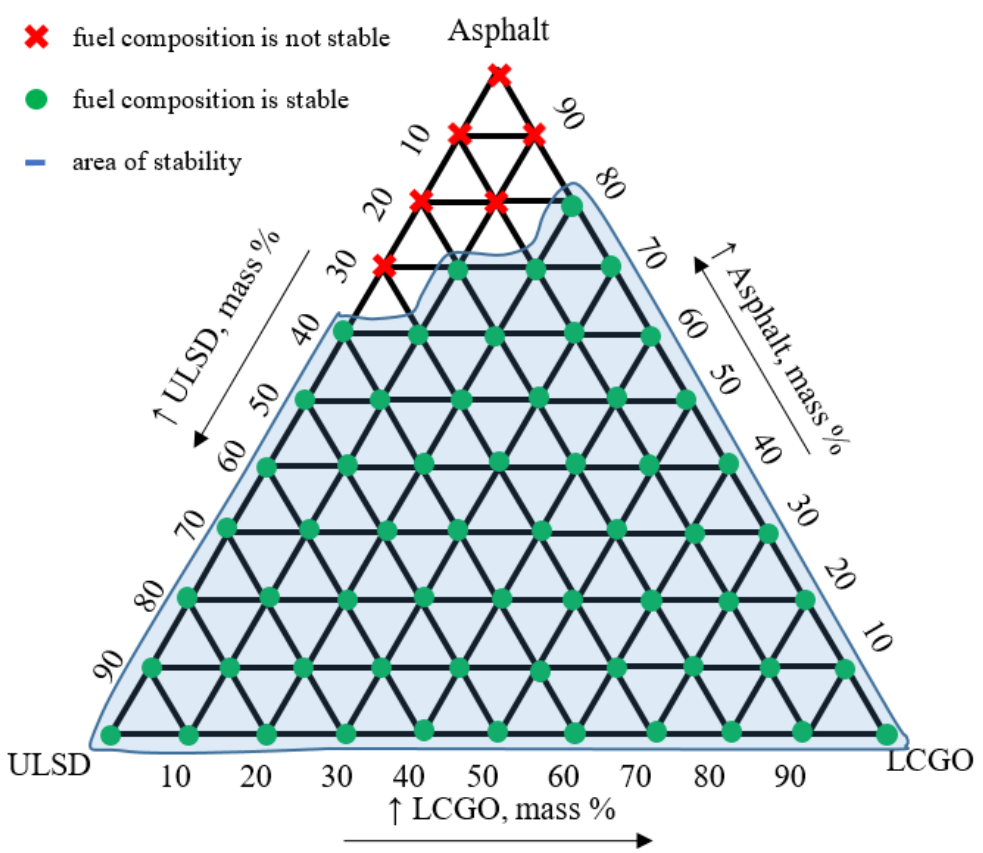

Fig. 2. The ternary phase diagram of the stability of the residual marine fuel Asphalt - ULSD LCGO.

Thus, the following components were taken as basic components for the preparation of residual marine fuel samples: visbreaker residue (asphaltene carrier), light gas oil catalytic creaking (aromatic hydrocarbon carrier), and hydrotreated straight-run diesel fraction (paraffin carrier).

Similarly to the first diagram, samples were prepared with different ratios of these components in increments of $10 \% \mathrm{wt}$. The total precipitate after aging was determined for each of the samples. The data obtained were plotted on a ternary phase diagram (unstable samples are indicated in red, stable samples are indicated in green).

Based on the data in table 1 , we can conclude that asphalt contains $3.2 \%$ wt. of asphaltenes, which can become coagulation centers and lead to loss of fuel stability with a change in hydrocarbon composition.

Asphalt, unlike, for example, VisRes, is not a product of the thermodestructive process and, along with asphaltenes, contains a large amount of resins $(28.4 \%$ wt.) and aromatic hydrocarbons $(59.7 \%$ wt.). Resins and arenas contribute to the stabilization of the fuel system, since they have a greater polarity. They are adsorbed on asphaltenes and form a solvate layer around the asphaltene particles.

Indeed, Figure 2 shows that the region of instability has decreased three times in comparison with the first ternary phase diagram (Figure 1). 
Mainly, such differences are due to the hydrocarbon composition of the residual component, i.e., asphalt. This is due to that asphalt contains compounds having large polarity (arenas and resins). In addition, asphaltenes of asphalt are of natural origin and have not been subjected to thermal degradation.

\subsection{The ternary phase diagram for residual marine fuel Asphalt - ULSD - LGODC}

As the need for oil residue processing increases, the capacities of the delayed coking process plants increase. The urgent issue is the use of gas oil fractions of this process.

The following components were taken as basic components for preparing residual marine fuel samples and constructing the third ternary phase diagram (Figure 3): asphalt (asphaltene carrier), light gas oil delayed coking (aromatic hydrocarbon carrier), and hydrotreated straight-run diesel fraction (paraffin carrier).

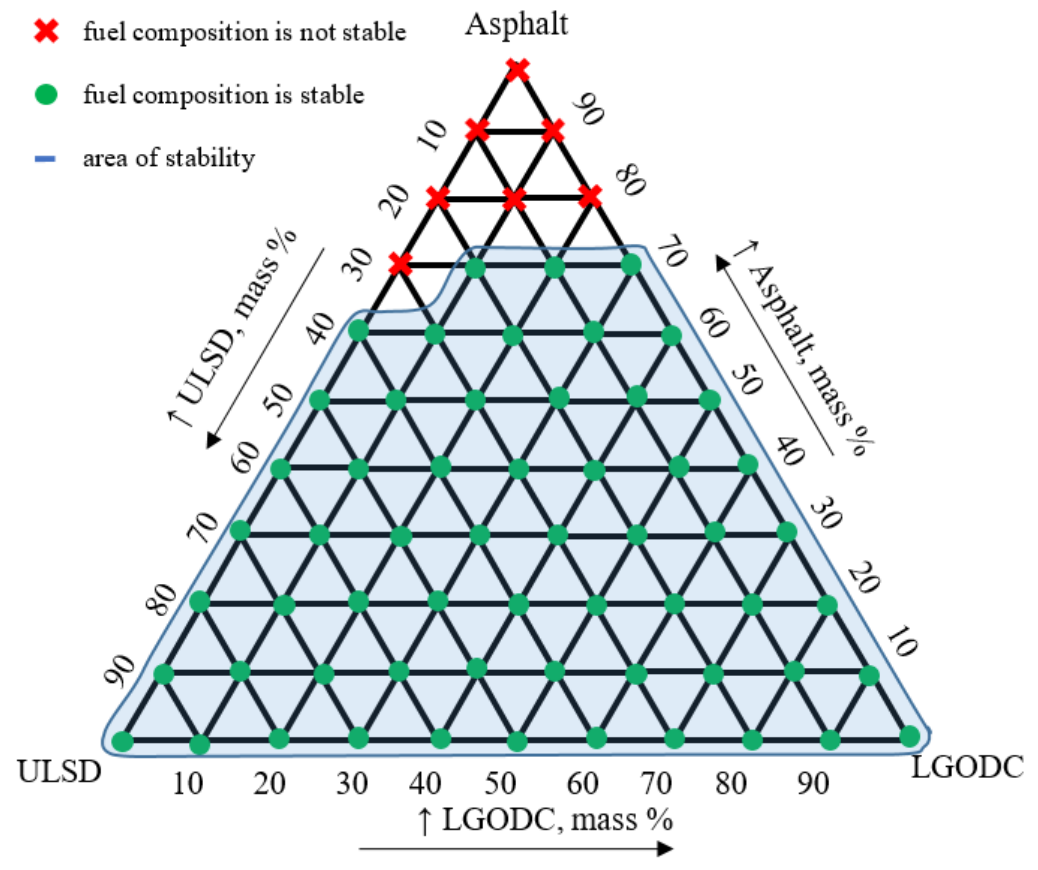

Fig. 3. The ternary phase diagram of the stability of the residual marine fuel Asphalt - ULSD LGODC.

Similarly to the first and second diagram, samples were prepared with different ratios of these components in increments of $10 \%$ wt. A total precipitate after aging was determined for each of the samples. The data obtained were plotted on a ternary phase diagram (unstable samples are indicated in red, stable samples are indicated in green).

Light delayed coking gas oil, as well as light catalytic cracking gas oil, is an aromatic hydrocarbon carrier component. In LGODC, it contains $37.52 \%$ wt. of aromatic hydrocarbons, in contrast to LCGO, where their content is two times higher $(75.23 \% \mathrm{wt}$.). The remaining two components are the carrier of paraffin-naphthenic hydrocarbons (ULSD) and the carrier of asphaltenes (asphalt) remaining unchanged compared to the second diagram.

When comparing Figures 2 and 3, we can conclude that the stability region differs slightly. Only a sample consisting of $20 \%$ wt. of asphalt and $80 \%$ wt. of LCGO is stable, 
and a sample consisting of $20 \%$ wt. of asphalt and $80 \%$ wt. of LCGO is unstable. Such differences are due to the hydrocarbon composition of LCGO and LGODC. Compared to LGODC, LCGO contains 2 times more arenes and 7 times less paraffin-naphthenic hydrocarbons. But since resins and arenas are contained in the asphaltene carrier component itself, this effect of the aromatic hydrocarbon carrier component has no strong effect on the stability of residual marine fuel. Thus, we can talk about the maximum concentration of aromatic hydrocarbons and resins in the fuel mixture (depending on the content of paraffin-naphthenic hydrocarbons and asphaltenes). At this concentration, all asphaltenes are dissolved in a dispersion medium. Therefore, adding more aromatic hydrocarbons will not affect the stability of the mixture.

\subsection{The graphic method for determining the stability of A.B. Stankiewiz}

Of all ternary phase diagrams presented in the work, the first diagram has the most complex nonlinear character of the dependence of the stability region on the component composition, since it contains VisRes (a product of light thermal cracking), which requires further consideration.

The laws of stability to separation were studied in the most detail for crude oil and oil mixtures based on it [18], since this is the most urgent task during storage and transportation $[19,20]$. One of the methods for predicting stability is Arthur B. Stankiewiz graphical stability criterion [16]. This method is based on determining the ratio of saturates, aromatic hydrocarbons, resins, and asphaltenes (SARA analysis). This method shows the most adequate results among graphical methods for predicting the stability of oil systems. In this method, the ratio of saturates / aromatic hydrocarbons ([S] / [Ar]) along the ordinate axis and the ratio of asphaltenes / resins ([A] / [R]) along the abscissa axis are plotted on the graph. Two parts of the graph are clearly marked as stable and unstable regions, separated by a metastability region (curve).

The results obtained when describing the stability of residual marine fuel by constructing a ternary phase diagram VisRes - ULSD - LCGO were compared with the results of the description of the stability of crude oil obtained using the Stankiewiz stability criterion.

The necessary calculations were carried out according to the additivity law for the ratio of saturated and aromatic hydrocarbons ([S] / [Ar]) and the ratio of asphaltenes and resins $([\mathrm{R}] /[\mathrm{A}])$ for each of the samples. The data obtained are used to build graphical dependencies.

For each of the samples of the first three-component phase diagram, the group hydrocarbon composition was calculated according to the additivity law:

$$
\mathrm{X}=\mathrm{XULSD} \cdot \omega \mathrm{ULSD}+\mathrm{XLCGO} \cdot \omega \mathrm{LCGO}+\mathrm{XV} \text { isRes} \cdot \omega \text { VisRes, }
$$

where $[\mathrm{X}]$ is the content of saturated $[\mathrm{S}]$, aromatic hydrocarbons [Ar], resins $[\mathrm{R}]$, asphaltenes [A] in each of the RMF samples, respectively; [X (ULSD)], [X (LCGO)], [X (VisRes)] is the content of [S], [Ar], [R], [A] in each of the RMF components, i.e., ULSD, LCGO and VisRes, respectively; $\omega$ (ULSD), $\omega$ (LCGO), $\omega$ (VisRes) are the contents of each of the components in the RMF.

The stability graph of the residual marine fuel (Figure 4) was obtained as follows:

1) All points $\mathrm{o}[\mathrm{S}] /[\mathrm{Ar}]$ and $[\mathrm{A}] /[\mathrm{R}]$ are plotted from the calculation according to formula (1) for fuel mixtures with a change in composition for each of the three components from 0 to $100 \% \mathrm{wt}$. in increments of $10 \% \mathrm{wt}$.

2) The extreme stability and instability points were identified. For clarity, the junction points are dashed lines, which have the same meaning as the liquidus and solidus curves in the phase diagrams of condensed liquid-solid systems: to the right (or above) of the line from the unstable points the fuel is unstable, i.e., this if "solid phase" (analogue of solidus), 
and to the left (or below) of the straight line from stable points, on the contrary, the fuel is stable, i.e., the "liquid phase" (analogue of liquidus).

3) The average values were found between two adjacent stable and unstable points, as shown in Figure 4, and for convenience, a dashed line, i.e., the metastability line, was connected.

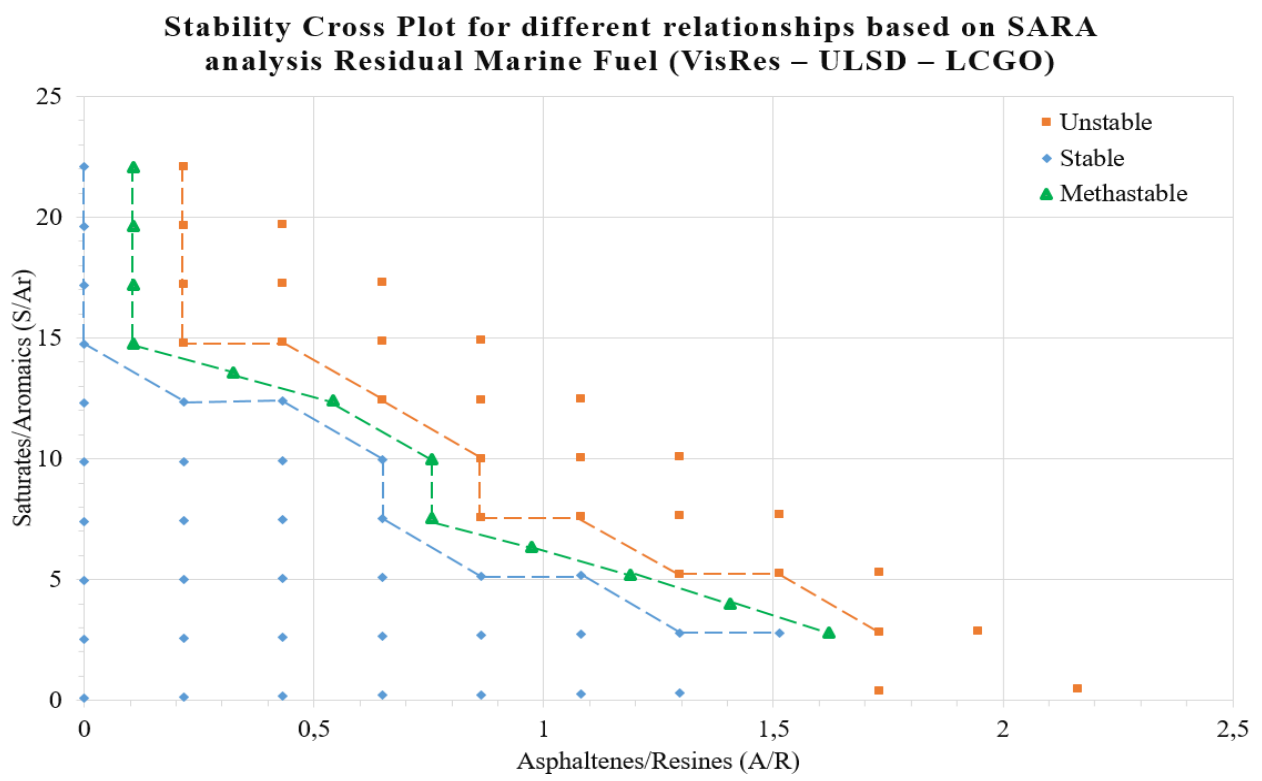

Fig. 4. Construction of lines of stability, instability and metastability of residual marine fuel VisRes ULSD - LCGO for the Stankiewics method.

Figure 5 shows a comparison of the stability graphs for residual marine fuel (VisRes ULSD - LCGO) and crude oil using the Stankiewicz method.

First of all, it is necessary to note the similar nature of the dependence of the obtained trend lines, described by the exponential law, both for crude oil [16] and for residual marine fuel. For residual marine fuel, the reliability coefficient of approximation (determination) $\mathrm{R}^{2}$ shows the degree of compliance of the trend model with the initial data and is equal to 0.9677. Therefore, equation (2) describes the model quite qualitatively:

$$
y=21.068 \mathrm{e}-1,199 x \text {, }
$$

where $\mathrm{y}$ is the ratio of saturated / aromatic hydrocarbon ([S] / [Ar]); $\mathrm{x}$ is the ratio of asphaltenes / resins ([A] / [R]).

The stability region is located below the curves (I - crude oil and III - marine residual fuel), the instability region is located above the curves (II - crude oil and IV - marine residual fuel), the curves according to the Stankiewicz method show a transient metastable state.

For residual marine fuel, the stability region takes up a large area due to the larger [S] / [Ar] ratio for equal amounts of $[\mathrm{A}] /[\mathrm{R}]$ compared to crude oil, despite the almost threefold increase in the $[\mathrm{A}] / \mathrm{R}]$ ratio. In addition, the estimated vertical segment of the metastability curve of marine fuel at low values of $[\mathrm{A}] /[\mathrm{R}]$ is located to the right of the same segment of crude oil. 


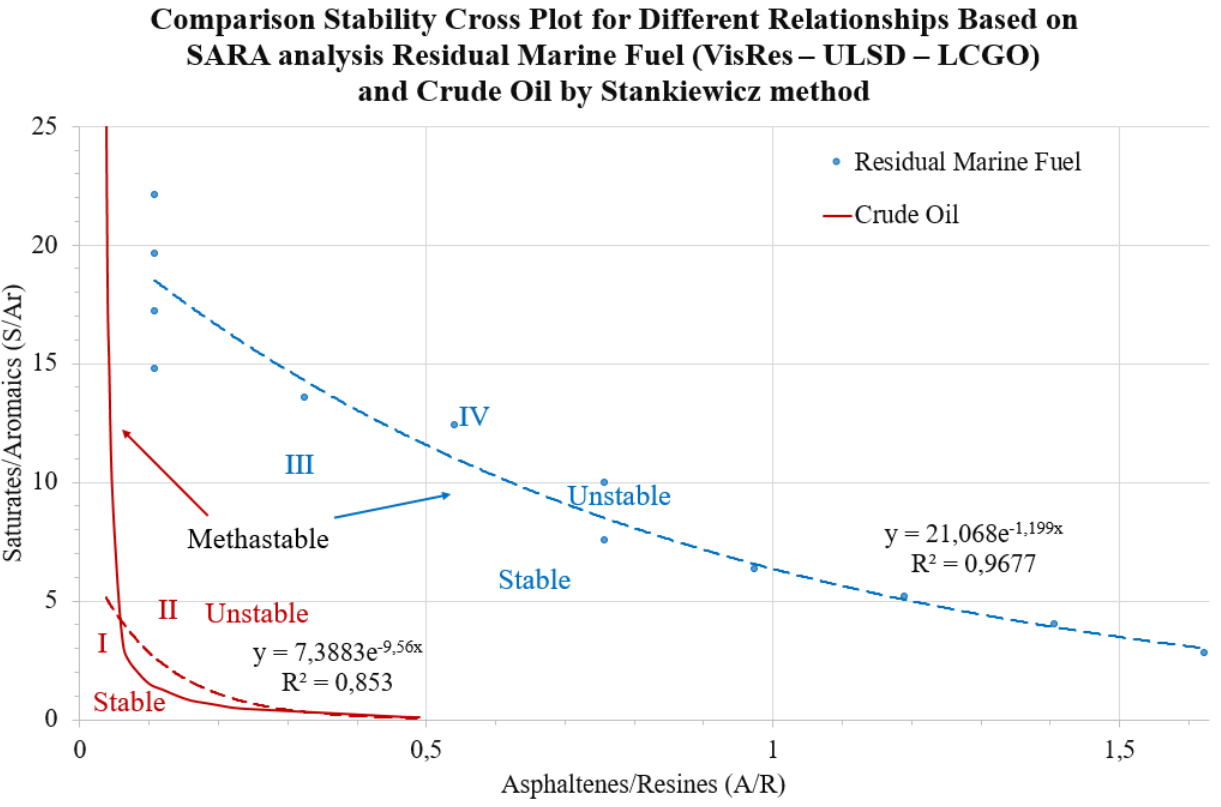

Fig. 5. Comparison of the stability graphs of crude oil and residual marine fuel VisRes - ULSD LCGO according to the Stankiewics method: I - the stability area of crude oil; II - the instability area of crude oil; III - the stability area of residual marine fuel; IV - the instability area of residual marine fuel.

Thus, the method proposed by Stankiewicz for describing the stability of crude oil can be used to describe the stability of compound residual marine fuels. This method, using the VisRes - ULSD - LCGO as an example, explains the complex nature of the dependence of the stability region through a metastability curve that obeys an exponential law and was obtained using SARA analysis.

\section{Conclusion}

A description method using a ternary phase diagram was developed to establish the dependence of the stability of the fuel system in residual marine fuels on their component and hydrocarbon composition.

Using ternary phase diagrams, stability regions of residual marine fuels based on VisRes - ULSD - LCGO, Asphalt - ULSD - LCGO, and Asphalt - ULSD - LGODC were found.

The ternary phase diagram of the VisRes - ULSD - LCGO has the greatest instability region, since the RMF samples contain asphaltenes of the thermodestructive process.

Arthur B. Stankiewicz graphic stability criterion was used to predict fuel stability based on VisRes - ULSD - LCGO. This method explains the complex nature of the dependence of the stability region through a metastability curve that obeys an exponential law and has been obtained using SARA analysis, and can be used to predict the stability of residual marine fuel.

This work was carried out as part of the State Assignment "Development of scientific foundations of innovative technologies for processing heavy hydrocarbon raw materials into environmentally friendly motor fuels and new carbon materials with controlled macro- and microstructural 
organization of mesophase". The study was conducted with the involvement of laboratory base of the Center for Collective Use of Saint Petersburg Mining University.

\section{Reference}

1. M.A. Nevskaya, S.G. Seleznev, V.A. Masloboyev, E.M. Klyuchnikova, D.V. Makarov, Environmental and Business Challenges Presented by Mining and Mineral Processing Waste in the Russian Federation. Minerals 9 (445):1-17 (2019).

2. A.E. Cherepovitsyn, F.D. Larichkin, A.G. Vorobiev, A. Ibrokhim, Economic prospects of the advancement in liquefied natural gas production. Gornyi Zhurnal (Mining Journal), 2 (2018).

3. V.M. Kapustin, E.A. Chernyisheva, E.N. Timin, R.V. Khakimov, New processes and technologies commercialized at the refineries of the Russian Federation. AIP Conference Proceedings. (2018).

4. Yu.A. Pakhomov, Yu.P. Korobkov, Ye.V. Dmitriyevskiy, G.L. Vasilyev, Toplivo $i$ toplivnyye sistemy sudovykh dizeley. (Moscow: RKonsult, 2004).

5. A.A. Alaseyeva, S.I. Khusnutdinov, S.M. Petrov, I. S. Khusnutdinov, A.G. Safiulina, N.Y. Bashkirtseva, Properties and Applications of Distillate Fractions from Highly Stable Dispersions of Liquid Pyrolysis Products. Chemistry and Technology of Fuels and Oils. 54(3): 271-277 (2018).

6. S.V. Budukva, P.M. Eletskii, O.O. Zaikina, G.A. Sosnin, V.A. Yakovlev, Secondary Middle Distillates and Their Processing. Petroleum Chemistry 59(9): 941-955. (2019).

7. K.L. Gawrys, P.M. Spiecker, P.K. Kilpatrick, The role of asphaltene solubility and chemical composition on asphaltene aggregation. Petroleum Science and Technology (21): 461-489. (2003).

8. J. Ancheta, HYDRO-MPC technology for heavy oil refining. Journal of Mining Institute (224): 229-234. (2017).

9. M.G. Ilyina, E.M. Khamitov, R.N. Galiakhmetov, I.A. Mustafin, A.F. Akhmetov, R.K. Shayakhmetova, A.G. Mustafin, Light gasoil of catalytic cracking: A quantitative description of the physical properties by joint use of chromato-mass-spectrometry and molecular dynamics. Journal of the Chinese Chemical Society 67(1): 33-40 (2020).

10. R.R. Sultanbekov, R.D. Terekhin, M.N. Nazarova, Effect of temperature fields and bottom sediments of oil products on the stress-strain state of the design of a vertical steel tank. Journal of Physics: Conference Series (1431): 1-7 (2020).

11. M.B. Vermeire, Everything you need to know about marine fuels. (Published by Chevron Global Marine Products. 2012).

12. A.D. Badikova, R.U. Muhamadeev, R.N. Shiryaeva, A.G. Mustafin, A.V. Rullo, I.G. Ibragimov, Spectral methods of analysis capabilities for investigation of the composition of oil sludges. SOCAR Proceedings, 4: 32-38 (2019).

13. K.D. Buchanan, Patent №0353851 US. Low-sulfur marine fuel. (2015).

14. T.N. N.K. Mitusova, Kondrasheva, Lobashova, M.M. Ershov, M.A. Rudko, V.A. Influence of Dispersing Additives and Blend Composition on Stability of Marine HighViscosity Fuels. Journal of Mining Institute, 228: 229-234 (2017).

15. T.N. Mitusova, N.K. Kondrasheva, M.M. Lobashova, M.A. Ershov, V.A. Rudko, M.A. Titarenko, Determination and Improvement of Stability of High-Viscosity Marine Fuels. Chemistry and Technology of Fuels and Oils, 53(6): 842-845 (2018). 
16. A.B. Stankiewicz, M.D. Flannery, N.Q. Fuex, G. Broze, J.L. Couch, S.T. Dubey, S.D. Iyer, Prediction of asphaltene deposition risk in E\&P operations. Third International Symposium on Mechanisms and Mitigation of Fouling in Petroleum and Natural Gas Production; 2002 March 10-14. New Orleans: AIChE. (2002).

17. N.K. Kondrasheva, V.A. Rudko, D.O. Kondrashev, V.S. Shakleina, K.I. Smyshlyaeva, R.R. Konoplin, A.A. Shaidulina, A.S. Ivkin, I.O. Derkunskii, O.A. Dubovikov, Application of a ternary phase diagram to describe the stability of marine residual fuel. Energy \& Fuel, 33: 4671-4675 (2019).

18. R. Guzman, Methods for determining asphaltene stability in crude oils. Fuel 188: 530543. (2017).

19. R.R. Sultanbekov, M.N. Nazarova, Determination of compatibility of petroleum products when mixed in tanks. Tyumen 2019. European Association of Geoscientists \& Engineers, 1, 1-5 (2019).

20. R.R. Sultanbekov, M.N. Nazarova, Studies of mixing high viscosity petroleum and pyrolysis resin to improve quality indicators. Scientific and Practical Studies of Raw Material Issues, 97-102 (2019). 